\section{Impact of anticholinergic burden on cognitive impairment, disability and malnutrition: a cross-sectional study among hospitalized older patients}

\author{
Mariapaola Nastri, Giulio Bartoli, \\ Paolo De Colle
}

Division of Geriatrics, Azienda Sanitaria Universitaria Integrata of Trieste, Trieste, Italy

\section{Abstract}

The anticholinergic burden (ACB) is known to be associated with the worsening of functional and cognitive status. This study aims at demonstrating a correlation between the ACB and the malnutrition, given the widespread effect on the digestive tract of anticholinergic medications. From 2012 to 2018, 2843 patients were recruited among the new admissions to our Geriatric Unit. For each patient the activities of daily living (ADL), the instrumental activities of daily living (IADL), the mini mental state examination (MMSE), the cumulative illness rating scale (CIRS), the mini nutritional assessment (MNA) and the ACB of medications were evaluated. The correlations between the ACB and the ADL $(\mathrm{P}<0.001)$, the IADL $(\mathrm{P}<0.001)$, the MMSE $(\mathrm{P}<0.001)$ scores were confirmed, and a significant correlation was also found between the $\mathrm{ACB}$ and the MNA $(\mathrm{P}<0.001)$ score. The CIRS and the ACB scores resulted to be independent predictors of all outcomes considered, in a linear regression model adjusted for age, sex, comorbidity and number of prescribed drugs. Therefore, ACB seems to have by itself an impact on physical and cognitive functions and on nutritional status.

\section{Introduction}

Polypharmacy (commonly defined as the assumption of more than 5 medications daily $^{1}$ ) has a great prevalence in the older population. It is a well-known trigger for many adverse outcomes, such as falls, hospitalizations, institutionalizations, development of delirium and dementia, and, ultimately, death. ${ }^{2,3}$ Multimorbidity may increase both the incidence of detrimental events and the number of prescribed medications. ${ }^{4}$ Nevertheless, also adverse drug reactions and drug-drug interactions may play an important role in determining these complications. ${ }^{5}$ Moreover, older patients are more susceptible to adverse drug reactions because of physiopatological alterations of metabolic pathways and pharmacokinetics. $^{6,7}$

Anticholinergic burden is a well-studied example of such detrimental effects of polypharmacy. Indeed, in association with the well-known anticholinergic effects, such as dry mouth, blurred vision, decreased gastrointestinal motility, urinary retention, and increased heart rate, many studies have proved an association between the long term use of anticholinergic drugs and other significant clinical outcomes, such as functional decline, cognitive impairment, rate of falls and hospitalizations, incidence of delirium, and mortality. ${ }^{8-10}$ Moreover, in addition to the medications employed to treat many medical conditions, such as Parkinson's disease, chronic obstructive pulmonary disease, urinary incontinence because of their anticholinergic activity, there are many drugs that have an unintended antagonistic effect on cholinergic receptors. ${ }^{8}$ Although the anticholinergic potency of many of these medications has been demonstrated only through in vitro studies and is pharmacodynamically weak if taken singularly, additive effects due to prescription of multiple anticholinergic drugs may be clinically relevant particularly in older patients, who have been demonstrated to be more susceptible to anticholinergic effects. ${ }^{11}$ For these reasons many drugs with anticholinergic activity are considered to be potentially inappropriate medications and the deprescribing is suggested. ${ }^{12}$

At the present time, there are no standardized tools to measure the total ACB of a single patient. Among all the instruments developed to evaluate it, the ACB scale is one of the most frequently used to demonstrate the adverse outcomes of the anticholinergic drugs. ${ }^{8}$ The multiform clinical presentation of the ACB is not surprising given the widespread distribution of the acetylcholine receptors, which can impair the functions of the central nervous system as well as the peripheral ganglia, including the gastrointestinal autonomic system. ${ }^{13}$ Although a possible causal relation between the ACB and the malnutrition may be hypothesized, the nutritional status has not been so far an object of research in the field of the ACB. This study aims to elucidate whether a correlation between the $\mathrm{ACB}$ and the malnutrition risk does exist, and whether it remains meaningful after the correction for age, sex, comorbidity and the total number of prescribed drugs.
Correspondence: Mariapaola Nastri, Division of Geriatrics, Azienda Sanitaria Universitaria Integrata of Trieste, Piazza dell'Ospitale 1,

Trieste, Italy.

Fax: +39.040.3992767

E-mail: nastrimariapaola@gmail.com

Key words: Anticholinergic burden; functional and cognitive impairment; malnutrition; polypharmacy.

Acknowledgments: we thank all residents of the Geriatric Unit for their precious contribution to perform the comprehensive geriatric assessment. We acknowledge Marco De Santis for database implementation.

Contributions: $\mathrm{MN}$ designed the study and revised the manuscript proof; GB made statistical analyses and wrote the first proof of the manuscript; PDC reviewed critically the manuscript.

Conflict of interest: the authors declare no potential conflict of interest.

Conference presentation: the abstract was awarded and presented orally during the XXXIII National Congress of SIGOT in Bologna on June $7^{\text {th }} 2019$.

Received for publication: 7 August 2019. Revision received: 29 October 2019. Accepted for publication: 7 November 2019

This work is licensed under a Creative Commons Attribution-NonCommercial 4.0 International License (CC BY-NC 4.0).

${ }^{\circ}$ Copyright: the Author(s), 2019

Licensee PAGEPress, Italy

Geriatric Care 2019; 5:8473

doi:10.4081/gc.2019.8473

\section{Materials and Methods}

We designed an observational retrospective study enrolling all subjects admitted to the Geriatric Unit of ASUITS, Trieste (Italy) from January $1^{\text {st }} 2012$ to December $31^{\text {st }} 2018$ who underwent a Comprehensive Geriatric Assessment (CGA). If the same patient had multiple readmissions, only the first one with complete data was considered.

\section{Comprehensive geriatric assessment}

Patients' functional status was assessed through activity of daily living (ADL) ${ }^{14}$, which were evaluated by the geriatrician at hospital admission, and instrumental activities of daily living (IADL) ${ }^{15}$, which were described by the patient himself or his relatives concerning the two weeks before the admission. The mini-mental state examination (MMSE) Italian version ${ }^{16}$ with correction for age and schooling was performed to 
evaluate the cognitive functions. The mini nutritional assessment (MNA) ${ }^{17}$ was calculated to evaluate the risk of malnutrition. The cumulative illness rating scale (CIRS) was calculated according to the standardized algorithm to assess the 13 items comorbidity burden (CIRSc13). ${ }^{18}$ A complete assessment of medications (including those prescribed on as-needed basis) was performed at the admission with the help of the patient and his caregiver (involving eventually the general practitioner, if both sources of information were unavailable or unreliable). The total number of drugs (including as-needed and topical medications) was recorded.

\section{Anticholinergic Burden scale}

The ACB scale assigns to each drug a score between 0 and 3 according to the estimated anticholinergic effect: 0 points are given to drugs with no known anticholinergic effect; 1 point is given to drugs with evidence of antagonist activity on muscarinic receptors from in vitro data; 2 points if there is clinical evidence of the drug anticholinergic effects in literature, prescriber's information, or experts' opinion of clinical practice; 3 points are assigned to those medications that may cause delirium according to literature, experts' opinion, or prescribers information. ${ }^{19,20}$ The total ACB score was obtained summing up the scores of all medications taken by a single subject before the admission to hospital.

\section{Statistical analyses}

Statistical analyses were performed with the free statistical software R. Spearman's rank correlation test was used to verify the correlations between the total ACB score and other variables of CGA. A linear model corrected for age, gender, comorbidity burden and total number of medications was used to test whether ACB score was an independent predictor of each CGA variable.

\section{Results}

During the period of the study 5440 subjects were admitted to our Geriatric Unit. There were 1028 multiple readmissions and 1569 patients with incomplete CGA. The final cohort therefore comprised 2843 subjects with median age 85.0 years (first and fourth quartile 79-90). Among these, 66.9\% were female. Polypharmacy had a prevalence of $47.8 \%$. Fully independent patients in all activities of daily living (IADL: 7-8) were $30.1 \%$ of total cohort. A complete physical dependency at admission (ADL: 0-
1) had a prevalence of $33.7 \%$. Mild cognitive impairment (MMSE comprised between 21 and 23.9) had a prevalence of $14.8 \%$; moderate cognitive impairment (MMSE between 11 and 20.9) had a prevalence of $27.6 \%$; severe cognitive impairment (MMSE less than 11) had a prevalence of $10.0 \%$. In the studied cohort $21.7 \%$ of subjects resulted malnourished (MNA<17), while $42.9 \%$ resulted at risk of malnutrition (MNA between 17 and 23.5). A statistical summary of CGA variables are reported in the Table 1. Mean ACB score was 0.93; an ACB score of 1 or more was achieved by $57.3 \%$ of the subjects of the cohort. The 20 most commonly prescribed drugs with anticholinergic properties are reported in the Table 2 along with their anticholinergic score, according to the ACB scale.

There was a significant correlation between the ACB score and the ADL $(\mathrm{P}<0.001, \mathrm{rho}=-0.11)$, the IADL $(\mathrm{P}<0.001$, rho $=-0.18)$, the MMSE $(\mathrm{P}<0.001$, rho $=$ $-0.07)$, and the MNA $(\mathrm{P}<0.001, \mathrm{rho}=-0.11)$ (Figure 1). In the age and sex adjusted linear regression model, CIRSc13 and $\mathrm{ACB}$ score resulted to be independent predictors of all outcomes considered, while the total number of prescribed drugs was an independent predictor of MMSE and ADL only. The complete results are displayed in the Table 3.

\section{Discussion}

In this study the existence of a direct correlation between the ACB of pharmacotherapy and the decline of functional and cognitive performances was confirmed in a cohort of hospitalized older patients. A significant correlation between the ACB and the risk of malnutrition has been found. Furthermore, it has been demonstrated that these correlations are not spurious, since they were driven not only by a higher number of drugs or by a higher comorbidity burden.

The ACB in the studied cohort was mainly driven by drugs with an ACB score equal to 1 (while drugs with $\mathrm{ACB}$ score equal to 2 or 3 were much rarely prescribed). Although these drugs have an anticholinergic effect demonstrated only in vitro, they seem to have an additive impact with significant clinical consequences.

It has also been highlighted that the number of prescribed drugs is not an independent predictor of IADL and MNA. On the contrary, the comorbidity assessment and the total ACB score resulted to be independent predictors of functional, cognitive and nutritional decline. These results suggest that clinicians should pay more atten- tion to these aspects of CGA than on the rough number of prescribed drugs.

This study has some limitations. First, although the ACB scale has been used in many European studies, ${ }^{21}$ it has been developed taking into account only drugs that are licensed in USA. Therefore, there may be some drugs with anticholinergic effects that are currently available in Italy, which have not been considered. Second, the ACB score gives only a rough estimation of the $\mathrm{ACB}$ of an individual patient because it does not make any distinction between

Table 1. Summary of the variables evaluated in the comprehensive geriatric assessment.

\begin{tabular}{lcc} 
Variables & $\begin{array}{c}\text { Mean } \\
\text { (standard } \\
\text { deviation) }\end{array}$ & $\begin{array}{c}\text { Median } \\
\text { (quartiles) }\end{array}$ \\
ACB score & $1.06(1.3)$ & $1(0-2)$ \\
ADL & $3.4(2.3)$ & $3(1-6)$ \\
\hline IADL & $4.0(3.0)$ & $4(1-7)$ \\
MMSE & $21.3(7.5)$ & $23.4(17.4-27.2)$ \\
\hline MNA & $20.8(5.5)$ & $22(17.5-25)$ \\
CIRS cl3 & $4.7(2.1)$ & $5(3-6)$ \\
\hline N of drugs & $5.6(3.1)$ & $5(3-8)$ \\
\hline
\end{tabular}

Table 2. The 20 most frequently prescribed medications with anticholinergic effect.

\begin{tabular}{lcc} 
Medications & ACB score & $\begin{array}{c}\text { Number } \\
\text { of patients }\end{array}$ \\
Furosemide & 1 & 913 \\
Warfarin & 1 & 323 \\
\hline Digoxin & 1 & 230 \\
Isosorbide & 1 & 210 \\
\hline Prednisone & 1 & 102 \\
Haloperidol & 1 & 83 \\
\hline Quetiapine & 3 & 76 \\
Atenolol & 1 & 69 \\
\hline Codeine & 1 & 66 \\
Paroxetine & 3 & 65 \\
\hline Alprazolam & 1 & 64 \\
Trazodone & 1 & 64 \\
\hline Metoprolol & 1 & 44 \\
Ranitidine & 1 & 34 \\
\hline Fentanyl & 1 & 32 \\
Cetirizine & 1 & 23 \\
\hline Diazepam & 1 & 22 \\
\hline Risperidone & 1 & 21 \\
\hline Venlafaxine & 1 & 19 \\
\hline Amitriptyline & 3 & 15 \\
\hline
\end{tabular}


drugs that are regularly taken and those taken as needed; furthermore, the scale does not account neither for the drug doses nor for the duration of the treatment (which can significantly affect the total anticholinergic burden). Moreover, this study did not assess patients' compliance to therapy. In spite of this, the results suggest that the ACB score is an instrument that is sensible enough to point out significant correlations with many relevant outcomes. Third, the generalizability of study findings to the whole older population is hindered by the recruitment of inpatients only, which are known to be characterized by a high prevalence of frailty if compared to older subjects living in the community. ${ }^{22}$ Finally, although the ACB score has resulted to be an independent predictor of development of cognitive, nutritional and functional impairment, more studies are needed to demonstrate that modifying the ACB can actually change the declining trajectories of these patients.

\section{Conclusions}

In conclusion, we demonstrated that the ACB negatively affects the functional, cog-

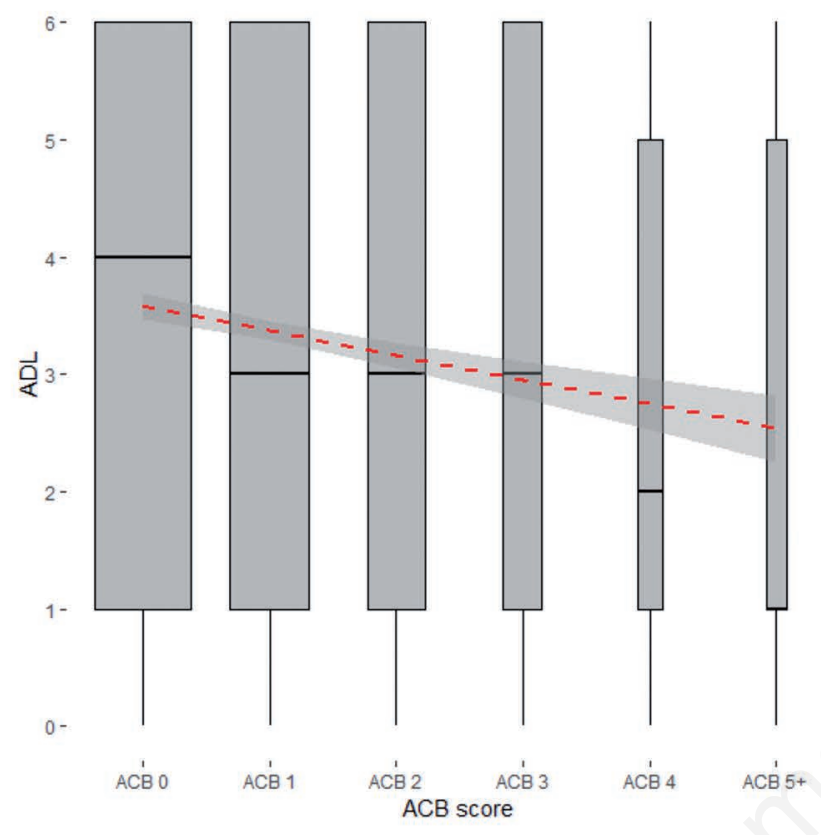

$\mathbf{A}$

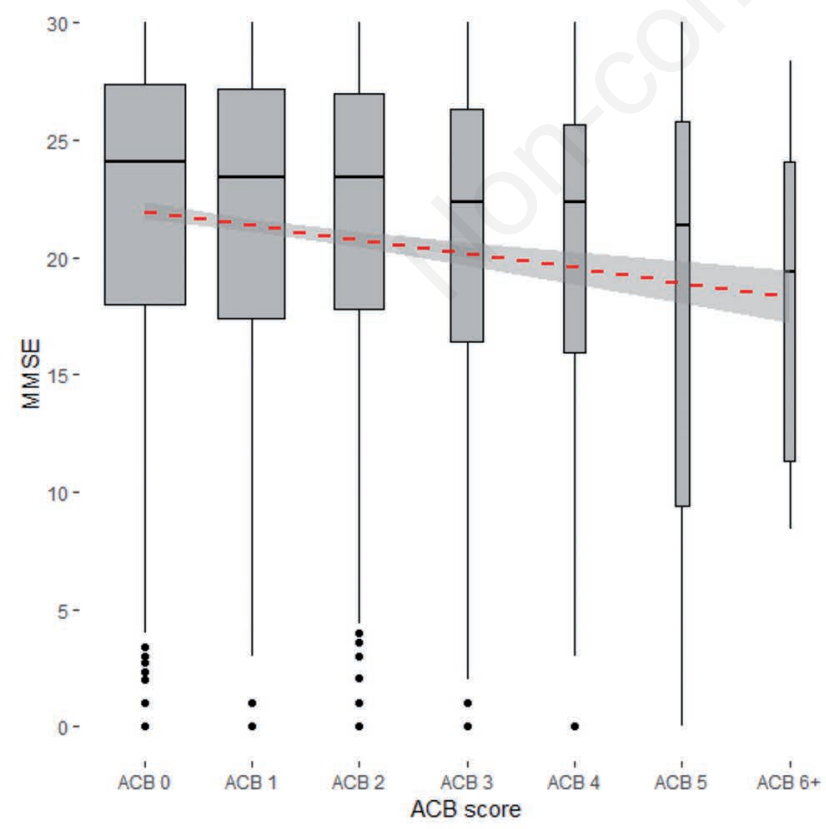

C

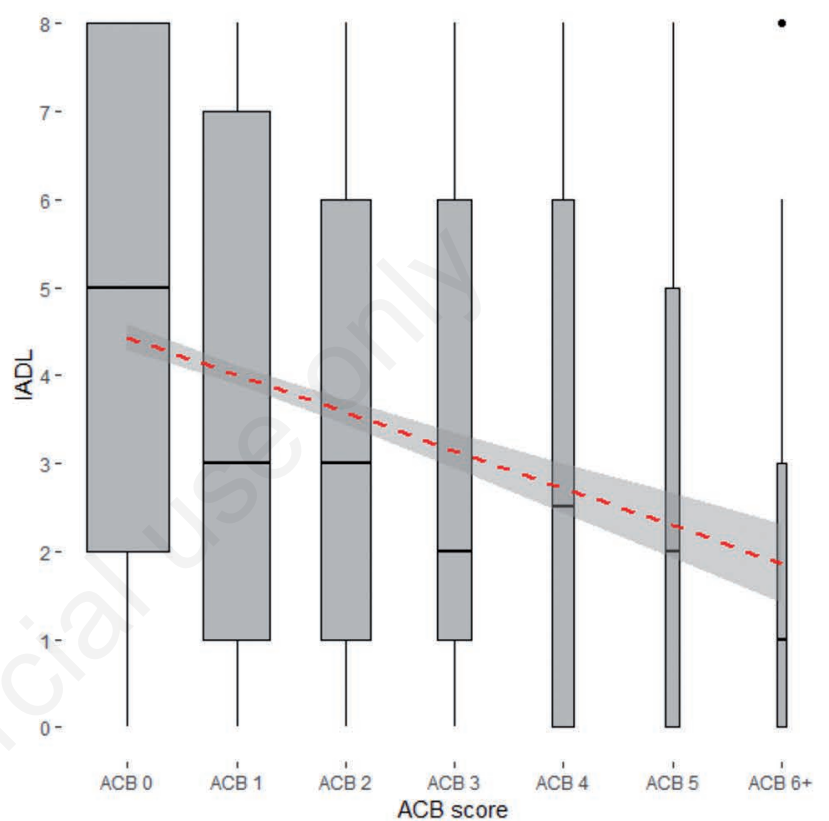

B

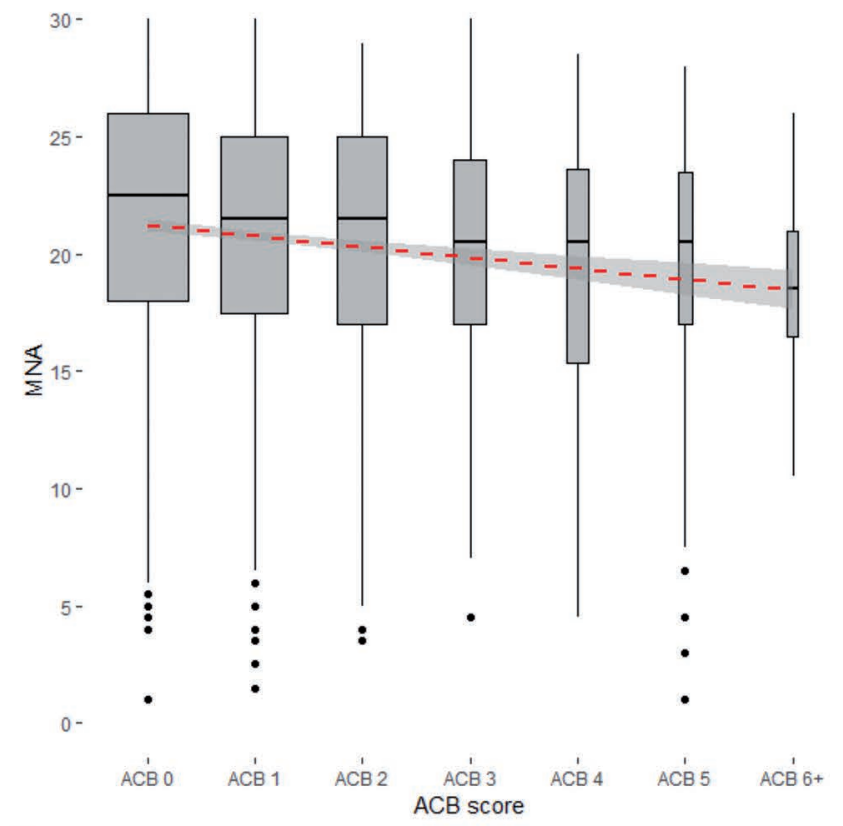

D

Figure 1. Correlation between ACB score and: A) activities of daily living (ADL); B) instrumental activities of daily living (IADL); C) mini mental state examination (MMSE); D) mini nutritional assessment (MNA). 
Table 3. Results of the age and sex adjusted linear models.

\begin{tabular}{|c|c|c|c|c|}
\hline & & Coefficient & Standard error & P-value \\
\hline Model 1 (for ADL) & $\begin{array}{l}\text { Age } \\
\text { Sex } \\
\text { CIRSc13 } \\
\mathrm{N} \text { of drugs } \\
\text { ACB score }\end{array}$ & $\begin{array}{c}-0.08 \\
0.42 \\
-0.30 \\
0.04 \\
-0.16\end{array}$ & $\begin{array}{l}0.01 \\
0.09 \\
0.02 \\
0.02 \\
0.04\end{array}$ & $\begin{array}{c}<0.001 \\
<0.001 \\
<0.001 \\
0.005 \\
<0.001\end{array}$ \\
\hline Model 1 (for IADL) & $\begin{array}{l}\text { Age } \\
\text { Sex } \\
\text { CIRSc13 } \\
\text { N of drugs } \\
\text { ACB score }\end{array}$ & $\begin{array}{c}-0.14 \\
-0.02 \\
-0.28 \\
0.01 \\
-0.34 \\
\end{array}$ & $\begin{array}{l}0.01 \\
0.11 \\
0.03 \\
0.02 \\
0.05\end{array}$ & $\begin{array}{c}<0.001 \\
0.88 \\
<0.001 \\
0.51 \\
<0.001\end{array}$ \\
\hline Model 1 (for MNA) & $\begin{array}{l}\text { Age } \\
\text { Sex } \\
\text { CIRScl3 } \\
\text { N of drugs } \\
\text { ACB score }\end{array}$ & $\begin{array}{c}-0.10 \\
0.94 \\
-0.50 \\
0.04 \\
-0.34\end{array}$ & $\begin{array}{l}0.01 \\
0.22 \\
0.05 \\
0.04 \\
0.09\end{array}$ & $\begin{array}{c}<0.001 \\
<0.001 \\
<0.001 \\
0.26 \\
<0.001\end{array}$ \\
\hline Model 1 (for MMSE) & $\begin{array}{l}\text { Age } \\
\text { Sex } \\
\text { CIRSc13 } \\
\text { N of drugs } \\
\text { ACB score }\end{array}$ & $\begin{array}{c}-0.20 \\
0.96 \\
-0.36 \\
0.39 \\
-0.95\end{array}$ & $\begin{array}{l}0.02 \\
0.29 \\
0.07 \\
0.05 \\
0.12\end{array}$ & $\begin{array}{c}<0.001 \\
0.001 \\
<0.001 \\
<0.001 \\
<0.001\end{array}$ \\
\hline
\end{tabular}

nitive and nutritional status, independently of polypharmacy and comorbidities. The prescription of the drugs with anticholinergic properties should be made with caution and the risk/benefits balance should be always checked. Physicians, in particular those who care for older people, should avoid prescribing those drugs with high ACB score and look for better pharmacologic or non-pharmacologic therapies to reduce anticholinergic side effects.

\section{References}

1. Masnoon N, Shakib S, Kalisch-Ellett L, Caughey GE. What is polypharmacy? A systematic review of definitions. BMC Geriatr 2017;17:230.

2. Fried TR, O'Leary J, Towle V, et al. Health outcomes associated with polypharmacy in community-dwelling older adults: a systematic review. J Am Geriatr Soc 2014;62:2261-72.

3. Bonaga B, Sánchez-Jurado PM, Martínez-Reig M, et al. Frailty, Polypharmacy, and Health Outcomes in Older Adults: The Frailty and Dependence in Albacete Study. J Am Med Dir Assoc 2018;19:46-52.

4. Wastesson JW, Morin L, Tan ECK, Johnell K. An update on the clinical consequences of polypharmacy in older adults: a narrative review. Exp Opin Drug Saf 2018;17:1185-96.

5. Rodrigues MC, Oliveira CD. Drug-drug interactions and adverse drug reactions in polypharmacy among older adults: an integrative review. Rev Lat Am Enfermagem 2016;24:e2800.

6. Gujjarlamudi HB. Polytherapy and drug interactions in elderly. J Midlife Health 2016;7:105-7.

7. Klotz U. Pharmacokinetics and drug metabolism in the elderly. Drug Metab Rev 2009;41:67-76.

8. Salahudeen MS, Duffull SB, Nishtala PS. Anticholinergic burden quantified by anticholinergic risk scales and adverse outcomes in older people: a systematic review. BMC Geriatr 2015;15:31.

9. Hsu WH, Wen YW, Chen LK, Hsiao FY. Comparative Associations Between Measures of Anti-cholinergic Burden and Adverse Clinical Outcomes. Ann Fam Med 2017;15:561-9.

10. Welsh TJ, van der Wardt V, Ojo G, et al. Anticholinergic Drug Burden Tools/Scales and Adverse Outcomes in Different Clinical Settings: A Systematic Review of Reviews. Drugs Aging 2018;35:523-38.

11. Fox C, Richardson K, Maidment ID, et al. Anticholinergic medication use and cognitive impairment in the older population: the medical research council cognitive function and ageing study. J Am Geriatr Soc 2011;59:1477-83.

12. 2019 American Geriatrics Society Beers Criteria ${ }^{\circledR}$ Update Expert Panel. American Geriatrics Society 2019 Updated AGS Beers Criteria ${ }^{\circledR}$ for Potentially Inappropriate Medication Use in Older Adults. J Am Geriatr Soc 2019;67:674-94.
13. De Vreese LP, Mantesso U, De Bastiani $\mathrm{E}$, et al. Anticholinergic burden in adult and elderly people with intellectual disabilities: Results from an Italian multicenter cross-sectional study. Publ Libr Sci One 2018;13:e0205897.

14. Katz S, Ford AB, Moskowitz RW, et al. Studies of Illness in the Aged: The Index of ADL: A Standardized Measure of Biological and Psychosocial Function. JAMA 1963;185:914-9.

15. Lawton MP, Brody EM. Assessment of older people: self-maintaining and instrumental activities of daily living. Gerontologist 1969;9:179-86.

16. Frisoni GB, Rozzini R, Bianchetti A, Trabucchi M. Principal life time occupation and MMSE score in elderly persons. J Gerontol 1993;48:S310-4.

17. Guigoz Y, Vellas B, Garry PJ. Assessing the nutritional status of the elderly: The Mini Nutritional Assessment as part of the geriatric evaluation. Nutr Rev 1996;54:S59-65.

18. Parmalee PA, Thuras PD, Katz IR, Lawton MP. Validation of the Cumulative Illness Rating Scale in a geriatric residential population. J Am Geriatr Soc 1995;43:130-7.

19. Boustani M, Campbell N, Munger S, Maidment I, Fox C. Impact of anticholinergics on the aging brain: a review and practical application. Aging Health 2008;4:311-20.

20. Aging Brain Program. Anticholinergic Cognitive Burden Scale 2012 Update. Available from: https:/www.uea.ac.uk/ documents/3306616/10940915/Antichol 
inergics/088bb9e6-3ee2-4b75-b8ceb2d59dc538c 2

21. Pfistermeister B, Tümena T, Gaßmann $\mathrm{KG}$, et al. Anticholinergic burden and cognitive function in a large German cohort of hospitalized geriatric patients. PLoS One 2017;12: e0171353.

22. O'Caoimh R, Galluzzo L, RodríguezLaso Á, et al. Prevalence of frailty at population level in European ADVANTAGE Joint Action Member States: a systematic review and metaanalysis Ann Ist Super Sanita 2018; 54:226-38 